An interesting story concerning the role of subunit interactions in aldolase has been spun by Chan and his colleagues. They first showed that the activity of the tetrameric enzyme is simply that of an assemblage of identical monomers, by preparing tetrameric hybrids of native and inactive succinylated subunits. The also devised the subterfuge of coupling the enzyme to a column matrix at, on average, a single subunit, and then dissociating the tetramer so as to leave only isolated monomers bound to the column. These proved fully active, though relatively prone to denaturation. It thus seems that there are certainly two (and presumably also intermediate) active forms of aldolase. Chan et al. (ibid., 2778) have now demonstrated that in the course of folding these coexist in the reaction mixture.

They have devised assays which will distinguish between the dissociated and tetrameric active forms. Discrimination is again achieved by the use of a critical urea concentration, in this case $2.3 \mathrm{M}$. In these conditions the tetramer is fully active, but the monomer unfolds. On the other hand, the instantaneous concentration of an active aldolase, monomeric and associated, in a mixture can be assayed by first introducing trypsin, which digests only the unfolded chains (or more precisely, because in optical terms refolding apparently precedes the appearance of enzymatic activity, the inactive population). Thus the kinetics of folding on transfer from concentrated guanidine hydrochloride into water can be followed by these two methods. The upshot is that total enzymatic activity appears according to a more or less first-order process, with no concentration dependence. In urea, by contrast, the kinetics show initially a curved profile, giving place to a lower linear rate, and are strongly concentration-dependent. The data give a convincing fit to a scheme whereby active monomers are first formed, and then associate to the urea-resistant tetrameric state. The identities of the urea-resistant and labile species were verified by determining the elution volumes of the two types of activity from a gel filtration column.

A striking artificial example of thermodynamic trapping of a native conformation by an association process is to be found in the work of Melchers and Messer (Eur. J. Biochem., 35, 380 ;

\section{Correction}

IN the editorial "Muscular Dystrophy and the Neurogenic Hypothesis" (Nature, 243, 258, 1973) the authors of the letter on page 287 in that issue were given as Kümpel and Dubowitz. The reference should, of course, have been to Gallup and Dubowitz.
1973), who are engaged in sifting the details of the process whereby antibodies to active wild type $\beta$-galactosidase of Escherichia coli encompass the activation of practically inactive mutant forms. Evidently, the affinity of the antibody for the conformation characteristic of the active state is sufficient to tilt the equilibrium between the active and inactive structures heavily towards the former. The activation occurs on a time scale of minutes, and is associated with an activation energy of about $23 \mathrm{kcal} / \mathrm{mole}$. There is no concentration dependence and evidently the conformational readjustment of the enzyme is the rate-limiting step.

EVOLUTION

\title{
Crossing the Barrier
}

from our Plant Ecology Correspondent

As new principles and concepts develop within each of the principal biological disciplines, so there is a tendency to apply these new ideas to the well-worn questions relating to the early development of life on Earth. Palaeontologists, biochemists and cell biologists have already contributed much to knowledge of early living systems; now it seems to be the turn of the ecologist to face some of these long-standing problems.

Stanley, for example, is an ecologist

\section{Sorting Out Signals}

Polypeptide chain initiation sites on messenger RNA can be isolated simply by forming test-tube initiation complexes with ribosomes and digesting away the unprotected regions of the RNA with ribonuclease. If the mRNA is appropriately labelled, the nucleotide sequence can be determined by any number of ingenious methods recently developed.

Hitherto such work has been confined to the small RNA viruses, $Q \beta$, R17 and $f 2$. Because DNA is not involved in the life cycle of these particular phage RNAs (unlike those of animal RNA viruses in general), the RNA must serve in other capacities besides that of directing protein synthesis: first, of course, the RNA must be replicated; second, control of protein synthesis (both temporal and stoichiometric) can occur only at the level of translation; third, folding of the phage RNA into the mature virus particle may introduce further constraints on the nucleotide sequences of RNA phage messengers in the regions of interest. The nucleotide sequences determining these various functions could conceivably overlap with the ribosome binding sites and be confused with polypeptide chain initiation signals.

Having pointed out the pitfalls in interpreting sequence data using the ribosome binding sites of RNA phage genomes alone, Arrande and Hindley announce in Nature New Biology next Wednesday (July 4) that they have now determined the sequence of the corresponding region of the polycistronic message (that which is transcribed by the host polymerase) for the "early" proteins of coliphage T7. The "early" genes are clustered at the left end of the T7 map and a single promoter site for the RNA polymerase has been identified by electron microscopy at the far left of this cluster. Arrande and Hindley have obtained a highly labelled "early" mRNA fragment of about 500 nucleotides by transcription of the phage DNA in vitro (a technique similar in principle to that used in their earlier work on R17 RNA). After digesting the labelled RNA-ribosome complex they were left with a piece of RNA thirty-eight nucleotides long whose sequence they analysed.

The only possible candidate (out of two) for the initiation triplet AUG for the first 9,000 molecular weight pro. tein corresponding to gene 0.3 is that situated at positions $19-21$ in the middle of the RNA fragment. This AUG is followed by the pentanucleotide sequence GAGGU which also occurs in the R17 A protein ribosome binding site. Moreover, the tetranucleotide GAGG appears in the corresponding region of the $\mathrm{Q} \beta$ genome. The conservation of the sequence AUGGAGG (U) is consistent with the (logical) prediction that the ribosome recognition site involves more than the initiation triplet AUG and its availability in a hairpin loop in the messenger RNA. The initiation site has to be distinguished from the triplet for internal methionine residues which is also AUG.

Examination of many more ribosome binding sites, both those within poly. cistronic messengers, those of both cellular and viral DNA-coded messengers, and those of animal RNA viruses which introduce DNA copies into the cell by means of reverse transcriptase, will be necessary before such vital processes as "modulation" (differential rates of translation of indivi. dual cistrons in a polycistronic message achieved by the recognition of specific nucleotide sequences by different initiation factors and/or ribosomes), the piracy of the cellular protein synthesiz. ing apparatus by infectious viruses, and other control mechanisms (for example, the choice between copying, replicating or translating RNA) can be explained (if not yet understood in terms of RNAprotein interactions). The fortunate nucleotide sequencers are going to have a field day sorting out the relevant signals. 